\title{
THE ROLE OF STAPHYLOCOCCAL SUPERANTIGENS IN THE PATHOGENESIS OF MARGINAL KERATITIS
}

\author{
D. G. R. JAYAMANNE ${ }^{1}$, M. DAYAN ${ }^{1}$, D. JENKINS ${ }^{2}$ and R. PORTER ${ }^{1}$ \\ Newcastle upon Tyne
}

\begin{abstract}
SUMMARY
Previous studies have demonstrated an increased incidence of delayed-type hypersensitivity to staphylococcal antigens in patients with blepharitis, but this does not predict subsequent development of marginal keratitis (MK). Superantigens are potent immunemodifying molecules produced by pathogenic organisms including Staphylococcus aureus. To study whether staphylococcal superantigens play a role in the development of $\mathrm{MK}$, conjunctival and lid margin cultures were taken from 26 subjects with MK and 24 controls. Four of 8 eyes with their first episode of MK grew strains of $S$. aureus, of which only one was superantigen-producing. None of the subjects with recurrent MK and only one control grew $S$. aureus. We conclude that staphylococcal superantigens are unlikely to play a central role in the development of MK. The absence of $S$. aureus on the lids of subjects with recurrent MK may reflect an alteration in lid flora due to previous topical antibiotic and steroid treatment.
\end{abstract}

The peripheral corneal infiltrates which develop in some patients with blepharitis (also termed marginal keratitis, catarrhal ulcers or marginal ulcers) are single or multiple curvilinear lesions in the peripheral anterior corneal stroma separated from the limbus by a rim of clear cornea and associated later with vascularisation from the limbus. The mechanism underlying the development of marginal keratitis (MK) in patients with chronic blepharitis is not fully understood. In at least a proportion of cases the presence of blepharitis has been attributed to exaggerated and abnormal bacterial colonisation of the lid margins ${ }^{1-3}$ and in 1946 Thygeson $^{4}$ isolated Staphylococcus aureus from the lids of patients with

From: ${ }^{1}$ Department of Ophthalmology and ${ }^{2}$ Department of Clinical Microbiology, Royal Victoria Infirmary, Newcastle upon Tyne, UK.

Correspondence to: Mr D. G. R. Jayamanne, FRCOphth, Department of Ophthalmology, Royal Victoria Infirmary, Queen Victoria Road, Newcastle upon Tyne NE1 4LP, UK. catarrhal ulcers, implicating this organism in its pathogenesis. In 1962, however, it was shown that the marginal ulcers themselves are sterile. ${ }^{5}$ An immune reaction to antigens present on the blepharitic lid margin is suggested by the observations that these ulcers are sterile, steroidresponsive and originate at the 10,2, 4 and 8 o'clock positions where the lids cross the corneal periphery in which antigen-presenting cells are most numerous. The development of MK has therefore been suggested to be due to delayed-type hypersensitivity to $S$. aureus antigens on the lids of patients with blepharitis.

The term 'superantigen' (SAg) was first coined in 1989 to describe a group of molecules produced by pathogenic organisms and capable of inducing activation, deletion and anergy ${ }^{6,7}$ in a large proportion of the $\mathrm{T}$ lymphocyte cell (and possibly B lymphocyte cell ${ }^{8}$ ) population.

SAg have been shown to be produced by the Gram-positive cocci $S$. aureus (e.g. toxic shock syndrome toxin-1 and enterotoxin B) and Streptococcus pyogenes (streptococcal pyrogenic exotoxins), by Mycoplasma arthritidis and by some retroviruses, and have been implicated in the pathogenesis of diseases as diverse as rheumatoid and mycoplasma arthritis, ${ }^{9}$ Kawasaki disease, toxic shock syndrome, scalded skin syndrome and the post-streptococcal autoimmune disorders. ${ }^{10}$

The aim of this study was to establish whether SAg-producing Staphyloccus aureus strains are present on the eyelid margins and conjunctiva of patients with MK, suggesting a role for these toxins in the pathogenesis of MK.

\section{PATIENTS AND METHODS}

Patients and controls were drawn from consecutive cases presenting to the eye department of Newcastle General Hospital over a 6 month period. The patients were diagnosed as having MK on the basis 
of clinical findings of single or multiple curvilinear lesions in the peripheral anterior corneal stroma separated from the limbus by a rim of clear cornea associated with blepharitis. No patient had experienced symptoms for longer than 7 days prior to swabs being taken. Patients with lid margin culturenegative and culture-positive swabs had been symptomatic for similar periods before swabs were taken.

All patients were treated with a combination of topical antibiotics and topical steroids. In none of the cases was the diagnosis changed on subsequent follow-up. Patients with MK were excluded from the study if they had previous unrelated corneal pathology, were contact lens wearers, or had recently received, or were currently receiving, topical or systemic antibiotics. In the MK group 8 of the 26 patients had no previous history of the disease. Nine of the remaining 18 patients were observed to have had one previous episode of MK. All other MK patients were observed to have had two or more episodes of MK. None of the patients with a past history of MK had received topical antibiotics or corticosteroids in the preceding 6 months.

Twenty-four age- and sex-matched controls were drawn from patients presenting to the eye department with non-corneal eye disease (e.g. cataract). None of the controls had a history or signs of acne rosacea.

In both patients and controls, both eyes (conjunctival and eyelid margins) were sampled with a cotton-tipped swab. Swabs were plated out onto horse-blood and chocolate agar plates. The swab was then broken off into Todd and Hewitt nutrient broth. Agar plates and broth were incubated at $37^{\circ} \mathrm{C}$ in a $5 \% \mathrm{CO}_{2} /$ air atmosphere for 18 hours. Ten microlitres of broth was sub-cultured onto blood agar which was incubated as above. Colonies of Staphylococcus aureus on agar plates were identified by Gram strain, catalase, Staphaurex and tube coagulase tests. All isolates of $S$. aureus were tested for enterotoxin A, B, C, D and toxic shock syndrome toxin-1 (TSST-1) by reverse passive latex agglutination (Unipath) by the Food Hygiene Laboratory, Central Public Health Laboratory, Colindale, London.

\section{RESULTS}

S. aureus was isolated from one of 24 control swab samples, but this was not a SAg-producing strain.
The only subjects from whom $S$. aureus was isolated in the MK group were those suffering their first episode of MK. Swab samples from 4 of the 8 patients $(50 \%)$ with their first episode of MK grew $S$. aureus. However, only one of the four isolates of $S$. aureus was SAg-producing. The SAg produced by the $S$. aureus isolate was TSST-1. The overall isolation rate for $S$. aureus in the MK patients was $15 \%$.

Conjunctival and eyelid margin swab results from the MK patients are summarised in Table I.

\section{DISCUSSION}

A role for cell-mediated hypersensitivity to $S$. aureus in the pathogenesis of MK was supported by the findings of Mondino et al. ${ }^{11}$ who produced a rabbit model of ulcerative blepharitis in which rabbits required a combination of both systemic (intradermal or subconjunctival) immunisation with $S$. aureus cell wall and repeated topical application of viable $S$. aureus to develop corneal infiltrates and phlyctenules. Rabbits that received topical $S$. aureus alone did not develop such lesions, suggesting that systemic immunity (and therefore possibly cellmediated immunity) to $S$. aureus is necessary for their development. It is worth noting, however, that less than $50 \%$ of the systemically immunised rabbits actually developed corneal infiltrates or phlyctenules.

In human subjects Ficker et al. ${ }^{12}$ found that 9 of 11 patients with a history of MK had enhanced cellmediated immunity to $S$. aureus and 8 of 11 to staphylococcal protein A. In a second study Ficker $e t$ $a l .{ }^{13}$ showed that enhanced cell-mediated immunity to $S$. aureus could be demonstrated in 46 of 116 (40\%) patients with blepharitis in the absence of antibodies to the staphylococcal antigen teichoic acid, but not among controls. However, the incidence of MK and the isolation rate of $S$. aureus from the lids were not significantly different in patients with and without enhanced cell-mediated immunity to $S$. aureus ${ }^{13}$ thus suggesting that the presence of delayed-type hypersensitivity to $S$. aureus is neither a prerequisite nor the sole explanation for the development of MK. Isolation of $S$. aureus from eyelids of normal subjects has been previously described. ${ }^{14}$

Although cell-mediated immunity to $S$. aureus does not appear to be present in all patients with $\mathrm{MK},{ }^{12,13}$ a cell-mediated immune response to anti-

Table I. Conjunctival and lid margin swab results from marginal keratitis (MK) patients

\begin{tabular}{|c|c|c|c|c|}
\hline No. of patients & Previous episodes of MK & $\begin{array}{l}S . \text { aureus isolated from } \\
\text { MK eye }\end{array}$ & $\begin{array}{l}S . \text { aureus isolated from } \\
\text { fellow eye }\end{array}$ & $\begin{array}{l}\text { Superantigen toxin } \\
\text { isolated (no.) }\end{array}$ \\
\hline 8 & None & 4 & 0 & TSST-1 (1) \\
\hline 9 & 1 & 0 & 0 & $(0)$ \\
\hline 2 & 2 & 0 & 0 & $(0)$ \\
\hline 7 & $>\overline{2}$ & 0 & 0 & (0) \\
\hline
\end{tabular}

TSST-1, toxic shock syndrome toxin-1. 
gens on the lids is suggested by the sterility, steroid responsiveness and location of these ulcers in the corneal periphery where antigen-presenting cells (APC) are most common.

Conventional antigens are processed and then presented to $\mathrm{T}$ cells in combination with major histocompatibility complex (MHC) molecules present on APC: class I MHC molecules are only expressed by APC and are recognised by cytotoxic T cells, whereas MHC class II molecules are present on all cells (except erythrocytes and trophoblasts) and are recognised by helper $T$ cells. The antigen specificity of a given $\mathrm{T}$ cell clone is determined by the unique combination of variable elements of its $T$ cell receptor $(\mathrm{V}$ and $\mathrm{J}$ of the alpha region and $\mathrm{V}, \mathrm{D}$ and $\mathrm{J}$ of the beta region). In contrast, SAg bind to the outer wall of the peptide-antigen binding groove on $\mathrm{APC}^{15}$ without being processed and are recognised by the $\mathrm{V}$ beta region of the $\mathrm{T}$ cell receptor alone. This means that all $\mathrm{T}$ cell subsets carrying a given $\mathrm{V}$ beta region are capable of recognising the same $\mathrm{SAg}$ and that binding is no longer MHC-restricted, with both cytotoxic and helper $\mathrm{T}$ cells responding to $\mathrm{SAg}$ presented by APC cells in this way. As there are only a few possible $\mathrm{V}$ beta sequences, up to $25 \%$ of all $\mathrm{T}$ cells are capable of recognising a given SAg as compared with approximately $0.1 \%$ capable of responding to a conventional antigen. ${ }^{16} \mathrm{SAg}$ have been implicated in localised inflammatory disorders such as rheumatoid arthritis, mycoplasma-induced arthritis, post-streptococcal glomerulonephritis and the scalded skin syndrome.

Our aim was to establish whether bacterial SAg from lid margin and conjunctival colonisation may play a part in the aetiology of MK. In this study, the isolation rate for $S$. aureus from eyelid margin and conjunctival swabs was $15 \%$. This compares with $25-27 \%$ in previous studies. ${ }^{2,13}$ Although S. aureus is believed to be a transient coloniser of the lid margin, the fact that both culture-positive and culturenegative patients had experienced symptoms for similar periods suggests that the absence of $S$. aureus on the lid margin in patients with recurrent MK may be significant. It is possible that patients with recurrent MK suffer chronic but variable lid margin colonisation with $S$. aureus, but it seems unlikely that we would fail to identify this in any of the patients with recurrent MK. However, all patients with a previous history of MK had received treatment with antibiotics and corticosteroids as part of their medication regime in the past, which may have altered the bacterial flora in these subjects.

It is interesting to note that the only subjects from whom we did culture $S$. aureus were those suffering their first episode of $\mathrm{MK}$, and that the isolation rate in these subjects was $50 \%$. Earlier reports have not documented different rates of isolation for $S$. aureus depending on the number of previous MK epi$\operatorname{sodes}^{2,13}$ and the significance of these findings in this small study is therefore unclear. Only one of four strains of $S$. aureus produced SAg toxins, which is in keeping with the observation that approximately $25-40 \%$ of all $S$. aureus strains produce SAg, ${ }^{17,18}$ and implies that there is no increase in the rate of superantigenic strains on the conjunctiva and eyelid margins of patients with MK. The numbers isolated were small, but while it is possible that we failed to identify some SAg-producing strains it is unlikely that all these patients were colonised with SAgproducing strains, suggesting that $\mathrm{SAg}$ production by $S$. aureus on the eyelid margins and conjunctiva is unlikely to be the primary aetiological factor in the development of MK.

A further study with increased numbers of subjects may be necessary to gain a picture of the toxins produced by the lid flora in isolated blepharitis. However, studies by Dougherty et al. ${ }^{2,3}$ have shown that $S$. aureus colonisation of the lids is uncommon in most types of blepharitis apart from staphylococcal and mixed staphylococcal/seborrhoeic blepharitis. This suggests either that the aetiology of MK is multifactorial and the presence of $S$. aureus on the eyelids is simply a contributing factor, or that $S$. aureus is not involved in the pathogenesis of MK. A multifactorial model of MK could be envisaged in which some blepharitis patients develop mechanical or bacterial lipase-induced ${ }^{11,19,20}$ damage to the cornea at sites where the corneal periphery is in contact with the inflamed lids, resulting in contact between APC and various bacterial antigens on the lids to produce an area of cell-mediated inflammation which spreads circumferentially and stimulates vascularisation if unchecked. At present the pathogenesis of MK and in particular the existence of a single triggering factor remains unclear. However, it remains a possibility that these corneal infiltrates represent the immune response to the presence of $S$. aureus toxins of various types on the eyelids of at least a sub-group of patients. It may be worth considering the in vitro response of patient's lymphocytes to the SAg and comparing this with controls in a future study.

We would like to thank Dr J. McLauchlin, Food Hygiene Laboratory, CPHL, London, for enterotoxin and TSST-1 testing.

Key words: Superantigens, Cornea, Eye disease, Marginal keratitis.

\section{REFERENCES}

1. Seal D, Ficker L, Ramakrishnan M, Wright P. Role of staphylococcal toxin production in blepharitis. Ophthalmology 1990;97:1684-8.

2. Dougherty JM, McCulley JM. Comparative bacteriology of chronic blepharitis. $\mathrm{Br} \mathrm{J}$ Ophthalmol 1984;68:524-8. 
3. McCulley JP, Dougherty JM. Bacterial aspects of chronic blepharitis. Trans Ophthalmol Soc UK 1986;105:314-8.

4. Thygeson P. Marginal corneal infiltrates and ulcers. Trans Am Acad Ophthalmol 1946;51:198-209.

5. Hogan MJ, Diaz-Bonnet V, Okumoto M, Kimura SJ. Experimental staphylococcic keratitis. Invest Ophthalmol 1962;1:267-72.

6. Miethke K, Wahl C, Heeg C, Wagner H. Superantigens: the paradox of T-cell activation versus inactivation. Int Arch Allergy Immunol 1995;106:3-7.

7. Ben-Nun A, Yossefi S. Staphylococcal enterotoxin B as a potent suppressant of $\mathrm{T}$ lymphocytes; trace levels suppress $\mathrm{T}$ lymphocyte proliferative responses. Eur $\mathbf{J}$ Immunobiol 1992;22:1495-503.

8. Zouali M. B-cell superantigens: implications for selection of the human antibody repertoire. Immunol Today 1995;16:399-405.

9. Fleischer B. Superantigens. APMIS 1994;102:3-12.

10. Herman A, Kappler JW, Marrack P, Pullen AM. Superantigens: mechanism of T-cell stimulation and role in immune responses. Annu Rev Immunol 1991;9:745-72.

11. Mondino BJ, Adamu SA, Pitchenkian-Halabi $H$. Antibody studies in a rabbit model of corneal phlyctenulosis and catarrhal infiltrates related to Staphylococcus aureus. Invest Ophthalmol Vis Sci 1991;32:1854-63.
12. Ficker L, Seal D, Wright P. Staphylococcal infection and the limbus: study of the cell-mediated immune response. Eye 1989;3:190-3.

13. Ficker L, Ramakrishnan M, Seal D, Wright P. Role of cell-mediated immunity to staphylococci in blepharitis. Am J Ophthalmol 1991;111:473-9.

14. Badiani D, Bron A, Elkington A, et al. Use of a novel transport method for the quantification of the normal flora of the external eye. Microb Ecol Health Dis 1988;1:57-9.

15. Leung DYM, Jeffery BT, Norris DA. The role of superantigens in skin disease. $\mathrm{J}$ Invest Dermatol 1995;105(Suppl):37s-42s.

16. Irwin MJ, Gascoigne NRJ. Interplay between superantigens and the immune system. J Leukocyte Biol 1993;54:495-501.

17. Lehn N, Schaller E, Wagner H, Kronke M. Frequency of toxic shock syndrome toxin and enterotoxin producing clinical isolates of Staphylococcus aureus. Eur J Clin Microbiol Infect Dis 1995;14:43-6.

18. Gittelman PD, Jacobs JB, Lebowitz AS, Tierno PM Jr. Staphylococcus aureus nasal carriage in patients with rhinosinusitis. Laryngoscope 1991;101:733-7.

19. Dougherty JM, McCulley JP, Silvany RE, Meyer DR. The role of tetracycline in chronic blepharitis. Invest Ophthalmol Vis Sci 1991;32:2970-5.

20. Shine WE, Silvany R, McCulley JP. Relation of cholesterol-stimulated Staphylococcus aureus growth to chronic blepharitis. Invest Ophthalmol Vis Sci 1993;34:2291-5. 